\title{
Bohmian trajectories for Kerr-Newman particles in complex space-time
}

\author{
Mark Davidson
}

Received: date / Accepted: date

\begin{abstract}
Complexified Liénard-Wiechert potentials simplify the mathematics of Kerr-Newman particles. Here we constrain them by fiat to move along Bohmian trajectories to see if anything interesting occurs, as their equations of motion are not known. A covariant theory due to Stueckelberg is used. This paper deviates from the traditional Bohmian interpretation of quantum mechanics since the electromagnetic interactions of KerrNewman particles are dictated by general relativity. A Gaussian wave function is used to produce the Bohmian trajectories, which are found to be multi-valued. A generalized analytic continuation (GAN) is introduced which leads to an infinite number of trajectories. These include the entire set of Bohmian trajectories. This leads to multiple retarded times which come into play in complex space-time. If one weights these trajectories by their natural Bohmian weighting factors, then it is found that the particles do not radiate, that they are extended, and that they can have a finite electrostatic self energy, thus avoiding the usual divergence of the charged point particle. This effort does not in any way criticize or downplay the traditional Bohmian interpretation which does not assume the standard electromagnetic coupling to charged particles, but it suggests that a hybridization of Kerr-Newman particle theory with Bohmian mechanics might lead to interesting new physics, and maybe even the possibility of emergent quantum mechanics.
\end{abstract}

Keywords Quantum Gravity · Kerr-Newman · Bohm · complex space-time · electron model · emergent quantum mechanics

PACS 03.65.Ta $\cdot 03.65 . \mathrm{Sq} \cdot 04.20 . \mathrm{Jb} \cdot 41.60 .-\mathrm{m}$

\section{Introduction}

This paper studies Kerr-Newman charged particles moving along free-particle Bohmian trajectories embedded in complex space-time. These are coupled directly to the electro-

Mark Davidson

Spectel Research Corp., Palo Alto, CA, USA

E-mail: mdavid@spectelresearch.com 
magnetic fields in the standard way, and the resulting Lienard-Wiechert potentials are analyzed. This form of coupling is dictated by the theory of general relativity. In the Bohmian interpretation of quantum mechanics, no such coupling of Bohmian particles to classical electromagnetic fields is assumed. Therefore, in this paper, I am deviating from the Bohmian interpretation, but using the Bohmian trajectories nonetheless. The Bohmian interpretation is perfectly consistent, and I do not mean by this to detract from it in any way. Rather I'm trying to see if there is anything to be learned about Kerr-Newman particles in this way. It appears that there might be.

Recently Maldacena and Susskind have proposed that quantum entanglement and the Einstein-Rosen bridge might be intimately related to one another [54,69]. They and others argue that gravity may be an emergent phenomenon, and that quantum entanglement is a crucial ingredient of this emergence 65, 24, 72. Einstein thought that quantum theory might possibly be derivable from general relativity and electromagnetism. This is clear from some of the statements in the Einstein and Rosen paper [32, as well as many other writings of Einstein dealing with unified field theory 67. The quest for a deeper understanding of quantum mechanics is still an active research topic. I cite only a small but significant sampling along these lines $71,66,73,61,62$. The results presented here touch upon these issues, and on the possibility of emergent quantum mechanics.

This paper is a follow-up to an earlier study [26,27] in which the complexified Lorentz-Dirac equation was analyzed, and by means of analytic continuation some of the runaway solutions became oscillatory, providing a mechanism for zitterbewegung, whose importance for the foundations of quantum mechanics has been emphasized 40, 29. The analytic continuation was justified by complex manifold techniques that have been prevalent in the Kerr-Newman metric literature [1,56, 55. In this paper, I extend these results by incorporating Bohmian trajectories for the Kerr-Newman particles. The idea that elementary particles might be Kerr-Newman singularities in general relativity has been boldly, eloquently, and persistently championed by Alexander Burinskii [18, 12, 13, 15, 20, 19. He also believes strongly in the connection between string theory and his Kerr-Newman electron theory, since the locus of singularities form one dimensional rings. The idea proposed here is that these particles may be traveling along Bohmian trajectories moving in complex space-time. Reviews of Bohmian mechanics are given in 4, 11,31,41. I want to develop a covariant theory here, and the original Bohm model, being non-relativistic, is not suitable. Although there are Bohmian models for the Dirac and Klein-Gordon equations in the literature, I have chosen for this first attempt a trajectory theory based on the relativistically covariant "proper time" wave mechanics of Fock and Stueckelberg 35, 68. An excellent review is the book by Horwitz 44. The application of Bohmian mechanics to this system was done by Kyprianidis and Fanchi in 46, 47,34. Holland has made some critical comments in 41, but I choose to work with it despite these, because the Stueckelberg approach allows separability in all four space-time axes, and this facilitates finding exact solutions needed for analytic continuation without errors. The problem of relativistic Bohmian mechanics was also considered in 30, and other approaches proposed there. Those could be considered in the future. In all of these Bohmian references there is no assumed coupling between the charged particles and the classical electromagnetic fields. In this paper I am borrowing the trajectories from the Bohmian interpretation, but then I'm deviating from the Bohm interpretation by treating the particles as standard Kerr-Newman particles in complex space-time which do couple to the classical electromagnetic fields. My initial interest in doing this was curiosity, but I would say that now the results obtained in 
this way are interesting. But it should not be taken as implying that the standard Bohmian interpretation of quantum mechanics should be modified by this, because it doesn't need such modification.

The null retarded and advanced time solutions determine the Liénard-Wiechert potentials in classical electromagnetism. In real Minkowski space, there are generally only two null solutions - one advanced and one retarded - for a given observation point and for a single classical timelike particle. In the complexified space-time there can be many complex-valued null solutions for a given trajectory, and the goal here is to study these. Since such particles would be very light, their gravitational distortion of the metric would be ignorable to a good approximation. But, the lingering effect that cannot be ignored is the complex space-time embedding. If there are multiple Riemann sheets for the Liénard-Wiechert potentials in complex space-time, as was found for example in [26], then this feature cannot be ignored, even when the metric distortions due to particle masses can be ignored. This fact has been largely overlooked in the physics literature because multi-valued fields and trajectories in complex space-time have not been given serious consideration previously 1 . So, in this paper, I shall work in complex Minkowski space, and consequently the mathematical description is tremendously simplified compared with curved metrics. But the lingering and critically important influence of gravity and general relativity is the complex space-time embedding, and the multi-valued Riemann sheet structure of both particle trajectories and their electromagnetic field interactions. These effects are not proportional to the gravitational constant, and can therefore affect measurements significantly in the small particle limit. Nevertheless, they can still be interpreted as gravitational effects.

The reader may reasonably ask if I'm proposing here that the complex space-time is actually an ontological reality. Personally, I'm agnostic on that point. One of the principle adherents of complex space-time approach, Ezra Newman, does not believe it's real, but that it's an extremely useful construct for mathematically analyzing the equations of general relativity for reasons that remain somewhat obscure [59. In section 11 I offer my own more detailed thoughts on this issue.

Kerr-Newman solutions are obtained most easily by considering point particles in complex Minkowski space. In all cases covered by the Kerr-Newman solutions (i.e. all values of mass, charge, and spin that define a solution), the particle is first considered as a structureless point particle. Spin arises by displacing the particle from the real axis. This is why I have chosen to begin here with the Klein-Gordon-Stueckelberg equation rather than a spin $1 / 2$ version, since spin implies some sort of internal structure to the particle, but the Klein-Gordon solution does not. It seemed like a good place to start. My ultimate goal is to try and describe the spin $1 / 2$ case as well, and I am studying this problem, but am not sure precisely how to proceed. I'm interested in possibly combining the results of [26], which gave a model for zitterbewegung within the KerrNewman framework, together with Bohmian trajectories. Alexander Burinskii has a different model for zitterbewegung which is based on a gravitating bag model involving additional field effects [17, 16], and he has argued that this leads to an explanation for Dirac equation. The bag structure gives internal structure to the particle, and it's no longer a simple point in complex space-time. I've chosen to work with the Stueckelberg formalism because it satisfies Wigner locality, and is manifestly Lorentz covariant,

1 The static Kerr-Newman solution to the electromagnetic field is double valued when the observation point is continued along paths in space-time. This is well known and studied. Here we are talking about multi-valued analytic functions of the world time variable when the trajectory is a function of this time and not static. 
and in addition the solutions are simpler because there is no mass shell constraint, and this allows separable solutions along all four space-time axes, without the mass constraint messing up separability. I wanted to work with exact closed-form solutions so that analyticity could be exactly studied, and analyticity cannot be studied with approximate solutions. This is a starting point. Future research can examine various ways to introduce spin. If this approach proves fruitful, then it may lead to a second application of Bohmian mechanics which links it to classical general relativity in a way that might point to a path to emergent quantum mechanics.

\section{Covariant Bohmian mechanics}

Following [46, 34, we first develop a Hamilton-Jacobi description of the many-particle covariant Stueckelberg equation in Minkowski space (metric signature [+,-,-,-] with $\mathrm{c}=1$ ). This version of relativistic mechanics, both classical and quantum, goes by other names such as Fock-Stueckelberg, proper time formalism, world or historical time formalism, Stueckelberg-Horwitz-Piron theory, covariant Schrödinger equation, etc. A fine recent review is given by Horwitz [44. It introduces an additional Lorentz invariant and universal world time, which is denoted here by the variable $s$. It plays a role similar to the proper time in relativistic classical mechanics, but it is not the same as proper time. The basic wave equation is a generalization of the many particle Schrödinger equation

$$
\begin{gathered}
i \hbar \frac{\partial \Psi}{\partial s}=K \Psi \\
K=\sum_{a=1}^{N} \frac{1}{2 m_{a}}\left(-i \hbar \frac{\partial}{\partial x_{a \mu}}-\frac{e}{c} A_{a}^{\mu}\right)\left(-i \hbar \frac{\partial}{\partial x_{a}^{\mu}}-\frac{e}{c} A_{a \mu}\right)+U\left(x_{1}, \ldots, x_{N}\right)
\end{gathered}
$$

where the momentum operator for particle $a$ is $p_{a}^{\mu}=-i \hbar \partial / \partial x_{a \mu}$. We follow the sign convention and metric signature used in 34] with no loss of generality.

The wave function $\Psi$ depends on a single Lorentz invariant "world time" parameter $s$ as well as the N-tuple of Minkowski coordinates $x_{a \mu}$. It is assumed to be a single valued function of the $x_{a \mu}$, at least when they are restricted to real values. $A_{a}^{\mu}$ is the external 4-vector potential acting on particle $a$. The extra potential $\mathrm{U}$ describes an additional non-electromagnetic potential term. All potentials are assumed to be analytic functions of all of their arguments. The normalization condition is

$$
\int \Psi^{*}\left(x_{1}, \ldots, x_{N}, s\right) \Psi\left(x_{1}, \ldots, x_{N}, s\right) d^{4} x_{1} \ldots d^{4} x_{N}=1
$$

In the standard Bohmian trajectory approach, followed in [34, one writes

$$
\Psi(x, s)=R(x, s) e^{i S_{B}(x, s) / \hbar}, S_{B}=\hbar \operatorname{Im}(\ln (\Psi))
$$

where for all $x_{a}$ and s real, $R$ and $S$ are real, $\rho=R^{2}$, and $R \geq 0$. The conservation equation is

$$
\frac{\partial \rho}{\partial s}+\sum_{a=1}^{N} \partial_{a \mu} V_{a}^{\mu} \rho=0, V_{a}^{\mu}=\frac{1}{m_{a}}\left(\partial_{a}^{\mu} S_{B}-\frac{e}{c} A_{a}^{\mu}\right)
$$


The vector potentials $A_{a}^{\mu}$ as seen by particle a will depend not only on $x_{a}$, but also in general on the other $N-1$ coordinates of the other interacting particles. These equations cannot be considered a complete description of the electromagnetic interaction, since the Liénard-Wiechert potentials for inter-particle interaction involve retarded (or maybe even advanced) times, and for relativistic particles such times depend not only on the particle positions but also on their entire trajectories for their calculation. But in quantum theory we do not have a trajectory to work with. One way to remedy this situation is to develop a second quantized field theory to handle the interactions, as is done in QED. This has led to a 5d version of electromagnetism called pre-Maxwell theory [48,49,51. If we try to use the Bohmian trajectories in real space time to calculate the electromagnetic fields produced, we quickly get unphysical results. For example, for a single free quantum particle, the Bohmian trajectories in general will be accelerated (and/or decelerated) by the quantum mechanical potential, and if they are charged then they will radiate electromagnetically and lose energy, but this clearly doesn't happen for free particles as Ehrenfest's theorem proves that they don't slow down in quantum mechanics, and experiments support this. In this paper, we consider the fields created by Bohmian trajectories, not in real, but in complex space-time where the radiation formulas are different. It is my hope that this will lead to new and interesting results, and perhaps allow the Bohm trajectories to be interpreted as classical sources for the electromagnetic fields. The reason that complex space-time makes a difference is that the Liénard-Wiechert potentials can have more than the two null-time solutions which they have in real space time. This comes about because of the possibility of multi-valued retarded times and even multi-valued trajectories in the complex case.

We can create a single particle current density by integrating the continuity equation over the other $\mathrm{N}-1$ particle positions. Suppose we want to integrate over all but particle a. We obtain

$$
\begin{gathered}
\rho_{a}\left(x_{a}, s\right)=\int\left(\prod_{m \neq a} d^{4} x_{m}\right) \rho\left(x_{1}, \ldots, x_{N}, s\right) \\
J_{a}^{\mu}\left(x_{a}, s\right)=\int\left(\prod_{m \neq a} d^{4} x_{m}\right) V_{a}^{\mu}\left(x_{1}, \ldots, x_{N}, s\right) \rho\left(x_{1}, \ldots, x_{N}, s\right)
\end{gathered}
$$

so the single particle continuity equation becomes

$$
\frac{\partial \rho}{\partial s}+\partial_{a \mu} J_{a}^{\mu}\left(x_{a}, s\right)=0, \partial_{a \mu} \int_{-\infty}^{+\infty} J_{a}^{\mu}\left(x_{a}, s\right) d s=0
$$

So we can identify the electromagnetic 4-current density for particle a by

$$
\mathcal{J}_{a \mathcal{E M}}^{\nu}(x)=q_{a} \int_{-\infty}^{+\infty} J_{a}^{\mu}\left(x_{a}, s\right) d s
$$

and where $q_{a}$ is a constant which is just the charge of the particle $Q_{a}$ up to a normalization factor.

$$
Q_{a}=\int d^{3} x \mathcal{J}_{a \mathcal{E} \mathcal{M}}^{0}(x)
$$


The Bohmian trajectory equation is the solution to the differential equation

$$
\frac{d x_{a}(s)^{\mu}}{d s}=\frac{1}{m_{a}}\left(\frac{\partial}{\partial x_{a \mu}} S_{B}(x, s)-\frac{q_{a}}{c} A_{a}^{\mu}(x)\right)
$$

One distinguishing characteristic of Fock-Stueckelberg wave mechanics is that the rest masses of particles are not fixed, but can change due to interactions. The off-mass-shell possibility in quantum theory has been analyzed in a series of papers by Greenberger [36, 37, 38, 39. The classical electromagnetic interaction alone cannot change the mass, but if the potential term $U\left(x_{1}, \ldots, x_{N}\right)$ is weak, then if the masses are initially on or near their mass shell values $m_{a}$, then as the world time variable $s$ advances, the mass shell constraint will remain approximately satisfied so that $m_{a} \approx p_{a}^{\mu} p_{a \mu}$, as described in 44. In this way the Stueckelberg theory includes the the Klein-Gordon equation as a limiting case when the masses can be taken as fixed. The advantage of the variable-mass Stueckelberg equation as opposed to the Klien-Gordon or other fixed mass equations is that Newton-Wigner locality holds for the position operators 44 while maintaining manifest covariance. It is also very similar to the Schrödinger equation, being first order in $s$ derivatives, and so many of the results of the usual Bohmian mechanics are easily carried over. The question of what draws particles back to the mass shell if they have somehow wandered off it is an area of active current research [43,50.

These functions $\Psi, R$, and $S_{B}$ in (4), defined for real arguments, are assumed to be sufficiently analytic locally to allow analytic continuation to all complex values for $x$ and $s$. This technique was used, for example, in $9,70,63,23 . R$ and $S$ can then become complex valued, and the resulting trajectories will trace out a curve in real space-time for real values of $\mathrm{s}$, and for complex values of $\mathrm{s}$, it will describe a $2 \mathrm{D}$ surface embedded in the $8 \mathrm{D}$ complex Minkowski space. The projection of this $2 \mathrm{D}$ surface onto the real hyperspace will look like a string, a point often emphasized by Burinskii [12].

\section{The free particle case}

$$
i \hbar \frac{\partial \Psi}{\partial s}=-\frac{\hbar^{2}}{2 M} \partial_{\mu} \partial^{\mu} \Psi=-\frac{\hbar^{2}}{2 M}\left[\partial_{0}^{2}-\partial_{1}^{2}-\partial_{2}^{2}-\partial_{3}^{2}\right] \Psi=\frac{\hat{p}^{\mu} \hat{p}_{\mu}}{2 M} \Psi
$$

We can consider a basis set of plane wave solutions, as usual, except that the mass is not constrained to be on the mass shell here

$$
\Psi(x, s)=\int \frac{d^{4} p}{(2 \pi \hbar)^{4}} \tilde{\Psi}(p, s) e^{i p^{\mu} x_{\mu} / \hbar}
$$

In general, $p^{\mu} p_{\mu} \neq M^{2}$. The operator for momentum is simply $\hat{p}^{\mu}=-i \hbar \frac{\partial}{\partial x_{\mu}}$. We have a straightforward uncertainty relation for each of the four coordinates, including a time-energy relation

$$
\sigma_{x^{\mu}} \sigma_{p^{\mu}} \geq \frac{\hbar}{2}, \mu \in\{0,1,2,3\}
$$

The rest mass associated with a plane wave solution is $m^{2}=p^{\mu} p_{\mu}$, with no limitation on the four independent values $p^{\mu}$. Negative energy particles are interpreted as moving backwards in time, which are in turn interpreted as anti-particles, an idea originally due to Stueckelberg which has long since been incorporated into quantum field theory. Negative $m^{2}$ particles are considered as tachyons. The question of how the particle mass avoids unobserved off-shell behavior is an area of current research [43,50,2]. These plane wave states are eigenstates for the $\hat{m}^{2}=\hat{p}^{\mu} \hat{p}_{\mu}$ operator. We say that if a state is built 
up by linear superposition from plane waves whose mass is given by $m^{2}=M^{2}$, then the particle is "on its mass shell". For all eigenstates of $\hat{m}^{2}$, the wave equation (12) becomes the standard Klein-Gordon equation by separating variables

$$
\Psi(x, s)=\psi_{m}(x) e^{-i s m^{2} / 2 M \hbar},\left[m^{2}-\hat{p}^{\mu} \hat{p}_{\mu}\right] \psi_{m}(x)=0
$$

A general state can be an arbitrary superposition of different mass plane waves. The "on-mass-shell" subset of wave functions do not form a complete set for the Hilbert space with inner product

$$
\langle\phi \mid \psi\rangle=\int \phi^{*}(x) \psi(x) d^{4} x
$$

For completeness, off-mass-shell states must be included.

\section{Bohmian description of the plane wave case}

$$
\psi(x)=A e^{i p^{\mu} x_{\mu} / \hbar}
$$

and so the Bohm-Hamilton-Jacobi function is, from (4) (up to an irrelevant additive function of $\mathrm{s}$ )

$$
S_{B}(x)=p^{\mu} x_{\mu}
$$

In this case, the velocity of the Bohmian trajectory is simply given by

$$
u^{\mu}=\frac{d x^{\mu}(s)}{d s}=\frac{\partial^{\mu} S_{B}}{M}=\frac{p^{\mu}}{M}
$$

and the Bohmian trajectory is simply given by $x^{\mu}(s)=u^{\mu} s+x^{\mu}(0)$. If the particle is on the mass shell, then $u^{\mu} u_{\mu}=1$, otherwise not. The plane wave is not normalizable, and so it's not a proper state for the Hilbert space. Note that the trajectory is single valued here as a function of $\mathrm{s}$.

\section{Gaussian wave functions and double-valued trajectories}

Holland 41] (in section 4.7) derives the Bohmian trajectory for a Gaussian wave function. We generalize his calculation to covariant Bohmian mechanics. For the nonrelativistic free particle Schrödinger equation, $i \hbar \frac{\partial \Psi}{\partial t}=-\frac{\hbar^{2}}{2 m} \nabla^{2} \Psi$, starting with the initial $(\mathrm{t}=0)$ wave function

$$
\Psi_{S}(\vec{x}, 0)=\left(2 \pi \sigma_{0}^{2}\right)^{-3 / 4} \exp \left(i \vec{k} \cdot \vec{x}-\vec{x}^{2} /\left(4 \sigma_{0}^{2}\right)\right)
$$

defining $\vec{u}=\frac{\hbar \vec{k}}{m}$ one obtains for time $\mathrm{t}$

$$
\begin{gathered}
\Psi_{S}(\vec{x}, t)=\left(2 \pi s_{t}^{2}\right)^{-3 / 4} \exp \left(i \vec{k} \cdot\left(\vec{x}-\frac{1}{2} \vec{u} t\right)-(\vec{x}-\vec{u} t)^{2} /\left(4 s_{t} \sigma_{0}\right)\right) \\
s_{t}=\sigma_{0}\left(1+i \hbar t / 2 m \sigma_{0}^{2}\right)
\end{gathered}
$$

The Bohmian action function is then 


$$
S_{B}(\vec{x}, t)=f(t)+\hbar \vec{k} \cdot\left(\vec{x}-\frac{1}{2} \vec{u} t\right)+\frac{(\vec{x}-\vec{u} t)^{2} \hbar^{2} t / 2 m \sigma_{0}^{2}}{4 \sigma_{0}^{2}\left(1+\hbar^{2} t^{2} / 4 m^{2} \sigma_{0}^{4}\right)}
$$

where $f(t)$ doesn't depend on $x$ and plays no role in determining the trajectory whose equation is

$$
\frac{d \vec{x}(t)}{d t}=\frac{1}{m} \nabla S_{B}(\vec{x}, t)=\vec{u}+\frac{(\vec{x}-\vec{u} t) \hbar^{2} t}{4 m^{2} \sigma_{0}^{4}\left(1+\hbar^{2} t^{2} / 4 m^{2} \sigma_{0}^{4}\right)}
$$

Holland finds the Bohmian trajectory for this system

$$
\vec{X}_{B}(t)=\vec{u} t+\vec{X}_{0}\left(1+\left(\hbar t / 2 m \sigma_{0}^{2}\right)^{2}\right)^{1 / 2}
$$

Next we generalize these results to the covariant Bohmian case. We have separability in the 4 Minkowski coordinate variables

$$
\Psi_{C B}(x, s)=\prod_{\mu=0}^{3} \psi_{\mu}\left(x^{\mu}, s\right)
$$

Despite this notation, $\Psi_{\mu}$ is not a Lorentz vector, but rather an indexed set of functions for a separation of variables. We will have a solution if the $x^{0}$ dependent wave function in this product satisfies the free particle equation (identical to the Schrödinger equation)

$$
i \hbar \frac{\partial \psi_{0}\left(x^{0}, s\right)}{\partial s}=-\frac{\hbar^{2}}{2 M} \frac{\partial^{2}}{\partial x^{02}} \psi_{0}\left(x^{0}, s\right)
$$

while simultaneously the $x^{j}$ dependent functions satisfy the complex conjugate equation.

$$
i \hbar \frac{\partial \psi_{j}\left(x^{j}, s\right)}{\partial s}=+\frac{\hbar^{2}}{2 M} \frac{\partial^{2}}{\partial x^{j}{ }_{j}} \psi_{j}\left(x^{j}, s\right), j \in\{1,2,3\}
$$

Using Holland's results, choose an initial (i.e. at $\mathrm{s}=0$ ) wave function of the form 26 with factors (no sum over $\mu$ here):

$$
\psi_{\mu}\left(x^{\mu}, 0\right)=e^{i k^{\mu} x_{\mu}}\left(2 \pi \sigma_{I \mu}^{2}\right)^{-1 / 4} \exp \left(-\left(x^{\mu}\right)^{2} /\left(4 \sigma_{I \mu}^{2}\right)\right), \mu \in\{0,1,2,3\}
$$

There are eight free real parameters $k^{\mu}$ and $\sigma_{I \mu}>0$, where $u^{\mu}=\frac{\hbar k^{\mu}}{M}$. These wave functions are normalized in $4 \mathrm{D}$. At other values of $s$ we have by analogy with the non-relativistic case 21)

$$
\begin{gathered}
\begin{array}{c}
\psi_{0}\left(x^{0}, s\right)=e^{i k^{0}\left(x_{0}-\frac{1}{2} u_{o} s\right)}\left(2 \pi \Sigma_{0}(s)^{2}\right)^{-1 / 4} \\
\times \exp \left(-\left(x^{0}-u^{0} s\right)^{2} /\left(4 \Sigma_{0}(s) \sigma_{I 0}\right)\right) \\
\psi_{j}\left(x^{j}, s\right)=e^{i k^{j}\left(x_{j}-\frac{1}{2} u_{j} s\right)}\left(2 \pi \Sigma_{j}(s)^{2}\right)^{-1 / 4} \\
\times \exp \left(-\left(x^{j}-u^{j} s\right)^{2} /\left(4 \Sigma_{j}(s) \sigma_{I j}\right)\right), j \in\{1,2,3\}
\end{array}
\end{gathered}
$$

where 


$$
\begin{gathered}
\Sigma_{0}(s)=\sigma_{I 0}\left(1+i \hbar s / M \sigma_{I 0}^{2}\right) \\
\Sigma_{j}(s)=\sigma_{I j}\left(1-i \hbar s / M \sigma_{I j}^{2}\right), j \in\{1,2,3\}
\end{gathered}
$$

it is convenient to also define

$$
\sigma_{\mu}(s)=\left|\Sigma_{\mu}(s)\right|=\sigma_{I \mu}\left(1+\left(\hbar s / 2 M \sigma_{I \mu}^{2}\right)^{2}\right)^{1 / 2}
$$

Although we use subscript notation for notational efficiency, this does not imply that $\sigma_{I \mu}, \sigma_{\mu}$, and $\Sigma_{\mu}(s)$ transform as Lorentz vectors.

$$
\begin{gathered}
\psi_{C B}(x, s)=e^{i k^{\alpha}\left(x_{\alpha}-u_{\alpha} s / 2\right)} \\
\times \prod_{\alpha=0}^{3}\left(2 \pi \Sigma_{\alpha}(s)\right)^{-1 / 4} \exp \left(-\left(x^{\alpha}-u^{\alpha} s\right)^{2} /\left(4 \Sigma_{\alpha}(s) \sigma_{I \alpha}\right)\right)
\end{gathered}
$$

and the Bohmian action (4) is

$$
S_{B}(x, s)=f(s)+\hbar k^{\alpha}\left(x_{\alpha}-u_{\alpha} s / 2\right)-\hbar \sum_{\alpha=0}^{3}\left(x^{\alpha}-u^{\alpha} s\right)^{2} \frac{\operatorname{Imag}\left(\Sigma_{\alpha}^{*}(s)\right)}{4 \sigma_{I \alpha}\left|\Sigma_{\alpha}(s)\right|^{2}}
$$

where $f(s)$ is a function only of s. These wave functions are not eigenstates for mass, energy, or momentum in general. If the $\sigma_{I \mu}$ values are all large, then the variance in the mass, energy, and momentum are all small, and so we can approximate an eigenstate of the mass with this. If in addition $u^{\mu} u_{\mu}=1$ then the particle is nearly an on-mass-shell eigenstate. Continuing by analogy with Holland's analysis [41, we obtain the Bohmian trajectories

$$
X_{B}^{\mu}(s)=u^{\mu} s+X_{B}^{\mu}(0)\left(1+\left(\hbar s / 2 M \sigma_{I \mu}^{2}\right)^{2}\right)^{1 / 2}
$$

and the s-velocity fields are given by

$$
\begin{gathered}
V_{B}^{\mu}(s)=u^{\mu}+X_{B}^{\mu}(0) \frac{s\left(\hbar / 2 M \sigma_{I \mu}^{2}\right)^{2}}{\left(1+\left(\hbar s / 2 M \sigma_{I \mu}^{2}\right)^{2}\right)^{1 / 2}} \\
=u^{\mu}+\frac{\left(X_{B}^{\mu}(s)^{\mu}-u^{\mu} s\right) \hbar^{2} s / 4 M^{2} \sigma_{I \mu}^{4}}{1+\left(\hbar s / 2 M \sigma_{I \mu}^{2}\right)^{2}}
\end{gathered}
$$

The mass is variable along this trajectory. It is given by the formula

$$
m^{2}(s)=M^{2} V_{B}^{\mu}(s) V_{B \mu}(s)
$$

Notice that the mass depends on $X_{B}^{\mu}(0)$, and that if this 4-vector is spacelike and very large, then $m^{2}(s)$ can become negative in principle. However, plugging plausible numbers into these formulas, this only occurs when $X_{B}^{\mu}(0)$ is many standard deviations from the mean, and I will ignore this complication here for the sake of brevity.

The trajectory function $X_{B}^{\mu}(s)$ is double-sheeted as a locally analytic function of a complex variable $s$ due to the square root factor in (37). We may write, for the trajectory function on the two Riemann sheets 


$$
\begin{gathered}
X_{B \pm}^{\mu}(s)=u^{\mu} s \pm X_{B}^{\mu}(0)\left(1+(\Gamma(\mu))^{2} s^{2}\right)^{1 / 2}, \Gamma(\mu)=\hbar / 2 M \sigma_{I \mu}^{2} \\
V_{B \pm}^{\mu}(s)=u^{\mu} \pm X_{B}^{\mu}(0) \frac{s(\Gamma(\mu))^{2}}{\left(1+(\Gamma(\mu))^{2} s^{2}\right)^{1 / 2}}
\end{gathered}
$$

The asymptotic behavior at large $\mathrm{s}$ is

$$
\begin{gathered}
V_{B \pm}^{\mu}(s)=u^{\mu} \pm X_{B}^{\mu}(0) \Gamma(\mu)+O\left(X_{B}^{\mu}(0) / \Gamma(\mu) s^{2}\right) \\
X_{B \pm}^{\mu}(s)=s\left(u^{\mu} \pm \Gamma(\mu) X_{B}^{\mu}(0)\right)+O\left(X_{B}^{\mu}(0) / \Gamma(\mu) s\right)
\end{gathered}
$$

the branch points are at $s= \pm i / \Gamma(\mu)$. The branch cut may be chosen to lie on any arbitrary curve joining these two branch points. Each of the two trajectories are timelike if $X_{B}^{\mu}(0)$ is close enough to the origin. For extremely large values of $X_{B}^{\mu}(0)$, the resulting trajectory can also be tachyonic or backward in time, depending on the orientation of $X_{B}^{\mu}(0)$. To set the scale for typical atomic problems, consider the value of $\Gamma$ for an electron with $\sigma_{I \mu}=1 \AA$ and $X_{B}^{\mu}(0)=1 \AA$. We find

$$
\Gamma(\mu)=5.7810^{15} s^{-1}, \text { and } X_{B}^{\mu}(0) \Gamma(\mu)=0.0019 c
$$

So, the velocities added by the quantum mechanical force are non-relativistic for this case. These two families of curves are timelike congruences, although they are not geodesics. In the case where all four $\Gamma(\mu)$ are equal, then they have a time dependent expansion, with the shear and twist both being zero in the language of timelike congruences 64.

Both Bohmian trajectories in (40) have acceleration. But, averaging the momentum of these two together yields

$$
P^{\mu}=\frac{1}{2} M\left(V_{B+}^{\mu}(s)+V_{B-}^{\mu}(s)\right)=M u^{\mu}
$$

which is a conserved value, and independent of $X_{B}^{\mu}(0)$, just as one would expect for a classical free particle. Moreover, the angular momentum for the pair of trajectories about the center point of the Gaussian at world time $s=0$ is

$$
\begin{gathered}
M_{ \pm}^{\mu \nu}(s)=X_{B \pm}^{[\mu} V_{B \pm}^{\nu]} \\
M^{\mu \nu}(s)=\frac{1}{2}\left[X_{B+}^{[\mu} V_{B+}^{\nu]}+X_{B-}^{[\mu} V_{B-}^{\nu]}\right]=0
\end{gathered}
$$

So the combined orbital angular momentum of the pair of trajectories equally weighted is zero in this case as well.

Why the double-valued solution is more general than just this one case Consider the Hamilton-Jacobi equation for Bohm action function $S_{B}$

$$
\frac{d S_{B}}{d s}+\frac{1}{2 M} \partial_{\mu} S_{B} \partial^{\mu} S_{B}-\frac{\hbar^{2}}{2 M} \frac{\partial_{\mu} \partial^{\mu} R}{R}+V=0
$$


Solve for one of the derivatives we find a double valued solution

$$
\begin{gathered}
\partial^{\alpha} S_{B}= \pm\left(-\eta^{\alpha \alpha} \sum_{\mu \neq \alpha} \partial_{\mu} S_{B} \partial^{\mu} S_{B}\right. \\
\left.+\eta^{\alpha \alpha} 2 M\left(\frac{d S_{B}}{d s}+\frac{1}{2 M} \partial_{\mu} S_{B} \partial^{\mu} S_{B}-\frac{\hbar^{2}}{2 M} \frac{\partial_{\mu} \partial^{\mu} R}{R}+V\right)\right)^{1 / 2}
\end{gathered}
$$

In general, the two Riemann sheets evidenced by the \pm sign can be visited by analytic continuation in the complex planes of the arguments of $S_{B}$. There is at least one exception. If the wave function is a perfect plane wave, then $\partial_{j} S_{B}$ is a constant, and has only one value. So, in general we can expect multi-valued trajectories in Bohmian mechanics in complex space time. In the case of free particles it is plausible then to expect the double-sheeted solutions of the form $\partial_{\mu} S_{B}(x, s)=M u_{\mu} \pm F_{\mu}(x, s)$ for some vector function $F_{\mu}$. There may be exceptions. The plane wave is an exception, but since it's not normalizable it's excluded from consideration.

\section{Generalized analytic continuation by partition of unity}

The possibility that multiple null roots contribute to solutions of the Liénard-Wiechert potentials suggests that when this occurs the solutions to Maxwell's equation are not unique. This was pointed out in 26] where a new form of generalized analytic continuation (GAN) was introduced. In that study, the trajectory function was an entire function of the complex proper time, although the retarded time was multivalued. In the present paper, we have found that the Bohmian trajectory function itself is not single valued as a function of complex world time $s$. This provides us with the option to apply GAN to the trajectories before calculating the set of null times. First we define exactly what is meant by GAN. Let $f(z, W)$ be a locally analytic function of a single complex variable $\mathrm{z}$ with a discrete set of branch points, poles, and essential singularities, and let $W$ be a set of complex parameters that $f$ depends on in a smooth and locally analytic way as well, and contained in some complex manifold. From the values of this function in any neighborhood of a point in the complex z plane, the function can be derived everywhere else in $\mathrm{z}$ by analytic continuation. If the function is multivalued, then this will be discovered by examining all analytic continuation paths. In general the function will have multiple Riemann sheets. Let their number be $N$, although $N$ could be infinite. $\mathrm{N}$ could depend on $W$, but we can take it to be an upper bound in this case without loss of generality. Now consider the following construction of a complex partition of unity. Let the $P_{i}(W)$ be smooth and analytic complex functions of the parameters $\mathrm{W}$ which satisfy the condition

$$
\sum_{i=1}^{N} P_{i}(W)=1,
$$

Where $N$ is the number of Riemann sheets of $f(z)$. Let branch cuts be selected which define the Riemann sheets uniquely. Next, consider a locally analytic point $\mathrm{z}$ which does not lie on any branch cut. Then, there exists a neighborhood $\mathcal{N}$ about z which 
does not intersect any branch cut. We define a new function. We start by doing nothing in the following form

$$
f(z)=\sum_{i=1}^{N} P_{i}(W) f(z)
$$

and next we create an intermediary function of $N$ variables $z_{i}$

$$
F\left(z_{1}, \ldots, z_{N}\right)=\sum_{i=1}^{N} P_{i}(W) f\left(z_{i}\right), z_{i} \in \mathcal{N}
$$

and finally we analytically continue each $z_{i}$ along a different path to arrive back at the starting value of $z$, but ending up on the ith Riemann sheet for the function $f$. In doing this, the paths for the various $z_{i}$ will leave the local neighborhood $\mathcal{N}$ temporarily as they must pass through branch cuts in order to reach other Riemann sheets. But eventually all the paths return to the starting point $z$.

$$
F\left(z_{1}, \ldots, z_{N}\right) \rightarrow \sum_{i=1}^{N} P_{i}(W) f_{i}\left(z_{i}\right) \rightarrow \sum_{i=1}^{N} P_{i}(W) f_{i}(z)=G\left(z,\left\{P_{i}(W)\right\}\right)
$$

This is what we mean by generalized analytic continuation or GAN. The new function $G\left(z,\left\{P_{i}(W)\right\}\right)$ depends on the arbitrary weighting parameters $P_{i}(W)$. So there is a great proliferation of one function into a whole family of GANs. Let us name this mapping GAN : $f(z) \rightarrow$ a subset of all locally analytic functions.

A note of caution The GAN continuation of a sum is not necessarily the same as the sum of the GANs. Some restrictions are required to avoid contradictions. For example, the function $\mathrm{f}(\mathrm{z})=0$ is single-valued, and we must insist that $G A N$ on this function yields just the zero function back again. But we can write $0=\sqrt{z}-\sqrt{z}$, and therefore if we assume linearity by writing $G A N(0)=G A N(\sqrt{z})+G A N(-\sqrt{z})$, where the addition would be the set of pairwise sums of all pairs of functions $f_{1}+f_{2}$ where $f_{1} \in G A N\left(f_{1}\right)$ and $f_{2} \in G A N\left(f_{2}\right)$. Obviously $G A N(\sqrt{z})+G A N(-\sqrt{z})$ contains many more functions than the zero function. So in general we cannot assume linearity for GAN. But there are some cases where linearity does not cause a problem. If one or both of the functions $A(z)$ and $B(z)$ are single valued, then we can safely write $G A N(A+$ $B)=G A N(A)+G A N(B)$. But in general we cannot. Similarly for multiplication. If $f(z)=A(z) B(z)$, then in general $G A N(f)=G A N(A B) \neq G A N(A) G A N(B)$ unless one (or both) of $\{A, B\}$ is an entire function in which case equality does hold. By $G A N(A) G A N(B)$ is meant just pairwise multiplication of the elements of $G A N(A)$ with those of $G A N(B)$.

\section{Examples}

For entire analytic functions, like $\exp (z)$ or any polynomial in $z$, the GAN continuation of the function gives just that single function back. There must be more than one Riemann sheet to give non-trivial results.

Lemma $\operatorname{GAN}(\sqrt{z})$ is the set of functions $X \sqrt{z}$, where $X$ is an arbitrary complex number.

Proof $\sqrt{z}$ has two Riemann sheets. Let $P_{1}+P_{2}=1$ with $P_{1}$ and $P_{2}$ complex numbers. The branch point is at $z=0$. We can draw the branch cut from this point to $\infty$ any way we choose. Once the branch cut is defined, the two Riemann sheets are then defined. It doesn't matter how we draw the branch cut and define the two Riemann 
sheets. The function $\sqrt{z}$ on the first Riemann sheet is the negative of its value on the second sheet. $f_{1}(z)=-f_{2}(z)$. Let $\mathrm{z}$ be a point on Riemann sheet $1 . G A N(\sqrt{z})$ is therefore $P_{1} \sqrt{z}-P_{2} \sqrt{z}=\left(P_{1}-P_{2}\right) \sqrt{z}$, but $P_{1}-P_{s}$ can take on any complex value. This function can be extended analytically in $\mathrm{z}$, and we get by so doing the original double-valued $\sqrt{z}$ function, but multiplied by an arbitrary factor $\left(P_{1}-P_{2}\right)$, and so the lemma is proven.

In like manner, it can be shown that the GAN of $z^{1 / n}$ is $X z^{1 / n}$, and of $\ln (z)$ is $X+\ln (z)$ for any complex $X$.

Now consider the family of Bohmian trajectories in our free particle example (37).

$$
X_{B}^{\mu}(s)=u^{\mu} s+X_{B}^{\mu}(0)\left(1+\left(\hbar s / 2 M \sigma_{I \mu}^{2}\right)^{2}\right)^{1 / 2}
$$

We require that $X_{B}^{\mu}(0) \neq 0$ for $\mu=0, . ., 3$. Note that $u^{\mu} s$ is single valued. In this case

$$
G A N\left(X_{B}^{\mu}(s)\right)=u^{\mu} s+X_{B}^{\mu}(0) G A N\left(\left(1+\left(\hbar s / 2 M \sigma_{I \mu}^{2}\right)^{2}\right)^{1 / 2}\right)
$$

This gives the set of all trajectories of the form

$$
G A N\left(X_{B}^{\mu}(s)\right)=\left\{u^{\mu} s+A^{\mu}\left(1+\left(\hbar s / 2 M \sigma_{I \mu}^{2}\right)^{2}\right)^{1 / 2} \forall A^{\mu} \in \mathbb{C}\right\}
$$

The Bohm trajectories, are the subset of these where $A^{\mu}$ are real.

$$
\operatorname{Re}\left(G A N\left(X_{B}^{\mu}(s)\right)\right)=\{\text { Bohmian trajectories }\}
$$

Where $\operatorname{Re}\left(G A N\left(X_{B}^{\mu}(s)\right)\right)$ is the subset of real trajectories. So we see that a single trajectory determines the whole set of Bohmian trajectories by the generalized analytic continuation procedure.

We see that all the Bohmian trajectories in this case belong to a GAN starting from a single seed trajectory which satisfies $X_{B}^{\mu}(0) \neq 0$ for any value of $\mu=\{0,1,2,3\}$. In other words if you take one of the Bohmian trajectories, you can derive all the other ones from it using the GAN procedure without knowing in advance what the wave function was. This result has been shown for the special case of the Gaussian wave function only. Whether it is a general result of the Bohmian theory for free particles is not known. Once you have analytic expressions for all the trajectories as functions of world time, you can then differentiate them to find the velocity and acceleration of these classical trajectories. If the external potential force is known, one can then calculate the quantum mechanical potential force for all of the trajectories as a function of position and world time. Using the velocities, one can also calculate Hamilton's principle function. Knowing both the quantum mechanical force and Hamilton' principle function, one should be able to figure out a class of equivalent wave functions that will give this Hamilton-Jacobi equation using Bohmian mechanics rules. Thus a single trajectory, in the cases studied in this paper at least, has hidden in its analytic structure information about all the other trajectories, and one never has to introduce wave mechanics as a separate postulate. This follows from the GAN procedure as outlined above. The single starting trajectory for this procedure is a purely particle picture, but the result is that a wave equation can be deduced from just this one trajectory which then generates all the other trajectories by the Bohmian mechanics rules. Proving this in general may be 
difficult, and it may not be generally true. I don't know how extensively these results can be extended to other cases. In the next few sections the electromagnetic fields in complex space-time are examined, and we shall see how this whole family of trajectories can contribute to the electromagnetic field produced by a charged particle moving along a single trajectory in this way. As far as the electromagnetic field produced is concerned, the source acts as a set or ensemble of trajectories, not just a single one. These conclusions are coming from a classical field theory, except that we put in the Bohmian trajectory by fiat. Everything being described here is therefore a classical phenomenon, yet it's very similar to aspects of quantum mechanics. The key ingredients are complex space-time, the Riemann sheet structure of multivalued functions, and the generalized analytic continuation.

\section{Electromagnetism in complex space-time}

The Kerr-Newman particle is modeled as a point charge which is slightly displaced from the real hypersurface in complex Minkowski space $\left(\mathbb{C} M^{4}\right)[57,58,55,56,14,15,53$, 60. The complex theory makes contact with reality by ultimately considering the electromagnetic fields "projected" onto the real space-time hyperspace. There are two equivalent formalisms that one can use. The Riemann-Silberstein complex vector field can be used 53

$$
\vec{W}=\vec{E}+i \overrightarrow{\mathrm{B}}
$$

or the equivalent covariant form 58

$$
W^{\mu \nu}=F^{\mu \nu}+i^{*} F^{\mu \nu},{ }^{*} W^{\mu \nu}=-i W^{\mu \nu}
$$

where ${ }^{*} W$ is the dual of $W$. From (59) we see that $W^{\mu \nu}$ is anti self-dual (ASD). The electromagnetic energy density and Poynting vector are $\left(\overrightarrow{\vec{W}}=C C\right.$ of $\left.\vec{W}=\vec{W}^{*}\right)$

$$
\mathcal{E}_{e l}=\frac{1}{2} \overrightarrow{\vec{W}} \cdot \vec{W}, \mathbf{P}_{e l}=\frac{i}{2} \vec{W} \times \overrightarrow{\vec{W}}
$$

The stress energy tensor on the real hyperspace is a function of the real-valued physical Faraday tensor

$$
4 \pi T^{\mu \nu}=F_{p h y s \lambda}^{\mu} F_{p h y s}^{\lambda \nu}+\frac{1}{4} g^{\mu \nu} F_{p h y s \alpha \beta} F_{p h y s}^{\alpha \beta}
$$

and this can be expressed in terms of the complex Faraday tensor with the substitution

$$
F_{p h y s}^{\mu \nu}(x)=\operatorname{Re} W^{\mu \nu}(x), \text { for all } x^{\alpha} \text { real }
$$

This simple prescription gets us back to real fields on real space-time. The absence of magnetic charge requires that $F_{\text {phys }}$ satisfies the electromagnetic Bianchi identity on real space-time, i.e. $\partial_{\mu}\left({ }^{*} F_{\text {phys }}\right)^{\mu \nu}=0$.

The static Kerr-Newman particle is modeled as a point charge located at a point in complex 3-space at $\mathbf{z}_{\mathbf{0}}=\mathbf{x}_{\mathbf{0}}+i \mathbf{b}$, where $\mathbf{x}_{\mathbf{0}}$ and $\mathbf{b}$ are real 3 -vectors. One introduces a complex Coloumb potential created by this particle

$$
\Phi(z)=q / \sqrt{\left(\mathbf{z}-\mathbf{z}_{0}\right)^{2}}
$$


and from this the fields can be derived, and they allow a metric of the Kerr-Schild type.

\section{Liénard-Wiechert potentials in complex spacetime}

The Liénard-Wiechert potentials are 45

$$
A^{\mu}(x)=\left.\frac{q v^{\mu}(s)}{v(s) \cdot(x-z(s))}\right|_{s=s_{r}}
$$

Normally only one retarded time contributes to the field from a single real timelike trajectory. The Faraday tensor from a single root may be calculated from the formula

$$
\begin{aligned}
F^{\mu \nu}(x)= & \frac{q}{V_{B}(s) \cdot\left(x-X_{B}(s)\right)} \\
& \quad \times\left.\frac{d}{d s}\left[\frac{\left(x-X_{B}(s)\right)^{\mu} V_{B}^{\nu}(s)-\left(x-X_{B}(s)\right)^{\nu} V_{B}^{\mu}(s)}{V_{B}(s) \cdot\left(x-X_{B}(s)\right)}\right]\right|_{s=s_{r}}
\end{aligned}
$$

Let $x^{\mu}$ be an arbitrary field point in real Minkowski space where we wish to calculate the electromagnetic field generated by the moving charged particle. Then the null condition for calculating the null world times is

$$
\left(x^{\mu}-X_{B}^{\mu}(s)\right)\left(x_{\mu}-X_{B \mu}(s)\right)=0
$$

Since we are working in complex space-time, we include all roots for the variable $s$ in the complex plane. This is a major difference between the complex space-time embedding and the usual real Minkowski space electromagnetism. The solutions to this equation are functions of the field point $\mathrm{x}$ which we take to be real. The multiple solutions to this equation will be denoted by $\left\{s_{n}(x)\right\}$. If $X_{B}(s)$ is a polynomial of degree $\mathrm{N}$ in $\mathrm{s}$, then there will be $2 N$ roots in the complex s plane obtained by standard analytical continuation. An example of this kind of multiple root situation was studied in 26] where there were an infinite number of roots.

If $X_{B \mu}(s)$ is multivalued as a function of $s$, as is the case for our Bohmian Gaussian example, then we can consider any root from any of the GAN continuation trajectories as possibly contributing to the field. We get the most interesting results if we apply GAN two times, first to the trajectories themselves, and next to the LiénardWiechert potentials. We look for all roots in the complex s plane, and there can be many such roots. In our case, we have just one retarded root per trajectory, assuming that only timelike trajectories are included in the Bohmian trajectory set, and therefore we include only these root 2 . So we now plug in the covariant Bohmian trajectories calculated above into this formula. For simplicity, let us choose all of the $\sigma_{I \mu}$ equal to each other. Let $\sigma_{I}$ denote the common value. Define a value of $\Gamma$ which is independent of $\mu$ as

$$
\Gamma=\frac{\hbar}{2 M \sigma_{I}^{2}}
$$

\footnotetext{
2 The non-timelike trajectories in the Bohmian set constitute a negligibly small fraction and are ignored here
} 
The fundamental trajectory equation is then

$$
X_{B}^{\mu}(s)=u^{\mu} s+X_{B}^{\mu}(0)\left(1+\Gamma^{2} s^{2}\right)^{1 / 2}
$$

where $X_{B}^{\mu}(0)$ is restricted to real values, and represents any one of the ensemble of Bohmian trajectories at $s=0$. So, the root equation for a single trajectory becomes (notation $\left.(x)^{2}=x^{\mu} x_{\mu}\right)$.

$$
\left(x-u s-X_{B}(0)\left(1+\Gamma^{2} s^{2}\right)^{1 / 2}\right)^{2}=0
$$

Applying a GAN transformation to the trajectory as in 56 and 57 we obtain

$$
\text { Re } G A N\left(X_{B}^{\mu}(s)\right)=\{\text { All real Bohmian trajectories }\}
$$

Although the GAN transformation produces complex trajectories as well as real ones, we ignore these by assuming that the weighting funtion has support only over real trajectories. Therefore we have one retarded time for each Bohmian trajectory, and they all contribute in a weighted sum to the electromagnetic field. Denote the solutions by $\left\{s_{r}\left(x, X_{B}(0)\right)\right\}$, and here $X_{B}(0)$ can be any real 4-vector. Each root solution produces a Liénard-Wiechert solution, and so we can write

$$
F^{u v}(x)=\sum_{X_{B}(0)} P\left(X_{B}(0)\right) F_{X_{B}(0)}^{\mu \nu}\left(x, s_{r}\left(x, X_{B}(0)\right)\right)
$$

where $F_{X_{B}(0)}^{\mu \nu}\left(x, s_{r}\right)$ is the field at point $x$ produced by a particle of charge $Q$ moving along a trajectory that passes through $X_{B}(0)$, and $P\left(X_{B}(0)\right)$ is the weight given to the trajectory that passes through $X_{B}(0)$ normalized so that 3

$$
\sum_{X_{B}(0)} P\left(X_{B}(0)\right)=1
$$

The summation in (71) is just a GAN transformation on the multivalued Faraday tensor. The solutions to Maxwell's equations in this case are simply not unique. In general the $P\left(X_{B}(0)\right)$ can be arbitrary complex numbers, but the natural choice for these is the Bohmian weighting which generates Born's rule, which becomes an integration over all starting positions $X_{B}(0)$ restricted to real values, and weighted by their probability density. $\rho=\Psi^{*} \Psi$ is the probability density. We can express it in terms of Bohmian trajectories

$$
\begin{gathered}
\rho(x, s)=\sum_{X_{B}(0)} P\left(X_{B}(0)\right) \delta^{4}\left(x-X_{B}(s)\right) \\
=\int d^{4} X_{B}(0) \rho\left(X_{B}(0), 0\right) \delta^{4}\left(x-X_{B}(s)\right)=\Psi^{*}(x, s) \Psi(x, s)
\end{gathered}
$$

and the integrated probability current is $J^{\mu}(x)=\int_{-\infty}^{\infty} V^{\mu}(x, s) \varrho(x, s) d s$ as in (7). The field is an ensemble average over all these trajectories

$$
F^{u v}(x)=\int d^{4} X_{0} \varrho\left(X_{0}\right) F_{X_{0}}^{\mu \nu}\left(x, s_{r}\left(x, X_{0}\right)\right)
$$

\footnotetext{
${ }^{3} \mathrm{Q}$ here is the actual physical charge of the particle
} 
The result is that the collective current density of the ensemble is proportional to the probability current calculated from the quantum wave equation. This is then equivalent to

$$
\partial_{\mu} F^{u v}(x)=\mathcal{J}_{\mathcal{E} \mathcal{M}}^{\nu}(x)=\frac{\hbar q}{2 M i} \int_{-\infty}^{\infty} d s \Psi^{*}(x, s) \overleftrightarrow{\partial^{\nu}} \Psi(x, s)
$$

and we see that the classical electromagnetic field can be produced by the coherent sum of all the Bohmian trajectories, and so it can look as if it's produced by an extended object. The reader should note that the integral of $\mathcal{J}_{\mathcal{E} \mathcal{M}}^{0}(x)$ over 3 -space is independant of $x^{0}$ because of (8) and the value of the parameter $q$ must be chosen so that this value gives the total charge of the particle. In words, a single charged particle trajectory generates a field whose generating current is that of an extended object. This is due to the non-unique solutions to the Liénard-Wiechert potentials when embedded in complex space-time, along with the particular solutions to the Bohmian trajectories which provide the mathematical justification, since any single one (excluding a set of measure zero) can be analytically continued (through GAN) to any other one, and thus a continuum of retarded times and positions are coherently added together yielding the resultant electromagnetic field of an extended object. This is an interesting result, but it should be remembered that it depends on applying GAN to the trajectories first to obtain an infinite set of them, and then applying it again to the Faraday tensor to produce a weighted sum over the multitude of retarded times so obtained. It is not obvious that solutions obtained in this way should be admitted in an electromagnetic theory in complex space-time. But if they are allowed, then we see that we can potentially obtain a solution to the ancient problem of the infinite electrostatic self-energy of classical point particles.

\section{The radiation problem in the full-relativistic case for a free particle}

The GAN continuation result provides a nice explanation for understanding why the free-particle Bohm trajectory need not radiate. Using results from 28 we see first of all that the Klein-Gordon current of a positive energy free-particle wave function are non-radiating electromagnetically. To make use of this, we can write any free-particle wave function $\psi$ as a sum over fixed mass eigenstates

$$
\Psi(x, s)=\int_{-\infty}^{\infty} d m \psi_{m}(x) e^{-i s m^{2} / 2 M \hbar},\left[m^{2}-\hat{p}^{\mu} \hat{p}_{\mu}\right] \psi_{m}(x)=0
$$

Now using (75), we can write the electromagnetic 4-current as

$$
\mathcal{J}_{\mathcal{E} \mathcal{M}}(x)=\frac{\pi \hbar^{2} q}{i} \int_{-\infty}^{\infty} d m \frac{1}{m} \Psi_{m}^{*}(x) \overleftrightarrow{\partial^{\mu}} \Psi_{m}(x)
$$

where $q$ is the charge up to a normalization factor, as explained in 9 and 10, It is necessary to assume that $\Psi_{m}=0$ for $m<\epsilon$ for some positive $\epsilon$. I assume that the $\Psi_{m}(x)$ are built up from positive energy plane waves which is a very good approximation to our Gaussian wave functions. Now, if two non-radiating currents are added together, then the sum is also a non-radiating current 28. Extending this argument, we see that $\mathcal{J}_{\mathcal{E} \mathcal{M}}(x)$ can be a non-radiating current provided that $\Psi_{m}(x)$ contains only positive mass and positive energy plane waves, and that certain other convergence conditions 
are satisfied as described in 28. Since $\Psi$ falls off as a Gaussian in all four coordinates, I expect that the convergence conditions will be satisfied. Strictly speaking, our Gaussian example does have an extremely small contribution from negative mass states, and so I'm assuming that these can be safely projected out without affecting the convergence requirements for the zero radiation proof.

So, if nature chooses to weight the trajectories by Bohm's prescription, then we get plausibly at least a non-radiating solution, and by this argument, we can think of each trajectory as being non-radiating. The only new ingredient is the idea of a generalized analytic continuation applied to complex space-time embedding. For the general free-particle case, it remains an open question.

\section{The near field for an isolated particle}

What does the electromagnetic field look like if we are near to the particle? This question introduces a measurement problem. How do we measure the near field of a quantum particle? One way is to scatter another charged particle off of it, and look at deep elastic scattering. But this involves solving a different quantum wave equation for the two-body problem. The near field of an isolated particle is not measurable, because as soon as we try to measure it we must introduce at least one new charged particle into interaction with it, and therefore it is no longer isolated. In the far field, we found that for an isolated charged particle in a particular quantum state the classical electromagnetic charge density and current could be taken proportional to the probability 4 -current derived from the wave function, and that this avoided radiation. This charge density is spread out over a large volume compared to say the Compton wavelength of the particle. It's not likely to produce any singular field points, although there may be exceptions. The point particle in complex space-time and moving along a Bohmian trajectory can therefore act like a kind of jellium of extended charge density in complex space-time. There is no obvious conflict with experiment if we simply assume that this extended charge model really does describe the reality of a completely isolated charged particle. Now if we make this assumption, which is based on the GAN continuation together with the assignment of the Bohmian weighting factors for each trajectory, then lo and behold, the classical electromagnetic energy and stress tensor is that of an extended charge which is no longer definitely singular, but calculable and probably for most wave functions finite due to the extended charge density. In other words, this theory offers a possible resolution to the long-standing problem of how to avoid the infinite self energy of an isolated point charge. In fact, since the extended charge density is non-radiating, it is concievable that the self-force of this charge density will be zero too, and therefore there may not be any runaway solutions that have plagued classical electromagnetism since the work of Abraham and Lorentz. I do not dismiss the Lorentz-Dirac equation though, because it may be the origin of zitterbewegung as discussed in 26. So, at least there is a possibility in this theory to resolve the ancient paradoxes of point particle electrodynamics which have plagued this theory for some time. These results bring to mind the theory of self-field electrodynamics by Barut and Dowling [5, 6, 8, 7].

An experiment has been proposed that could test this jellium picture of an isolated quantum charged particle. It assumes that in the presence of a weak classical external electromagnetic field, the extended charge distribution (75) is still valid. This hydrodynamic model leads to possibly testable bremsstrahlung effects [25]. 
8 In the non-relativistic limit, the radiation fields of the two Riemann sheets cancel if we average them together

In the non-relativistic limit, we can show the lack of radiation in another way that does not involve the assumption of GAN. In this limit we have

$$
u=\left\{u^{0}, \vec{u}\right\}, \vec{u}^{2} \ll\left(u^{0}\right)^{2}, \text { and } s_{r+} \approx s_{r-}
$$

and therefore from (40), (41), and $V_{B-}^{\nu}(s) \approx V_{B+}^{\nu}(s)$ it follows that the radiation field from just these two sources vanishes if they are weighted equally.

$$
F^{\mu \nu}(x)=\frac{F_{+}^{\mu \nu}(x)+F_{-}^{\mu \nu}(x)}{2}=0+O\left(\frac{1}{R^{2}}\right)
$$

And so the total electromagnetic radiation is zero in this case also, but only standard analytic continuation is required, not the more radical GAN technique. Complex spacetime is still required. The radiation fields of the two particles cancel one another in the non-relativistic limit. So, we have a second possible way of explaining why free Bohmian particles might not radiate, or radiate very much, in the non-relativistic limit. Both Bohmian trajectories in (40) experience non-zero acceleration. However, averaging the momentum of these two together yields

$$
P^{\mu}=\frac{1}{2} M\left(V_{B+}^{\mu}(s)+V_{B-}^{\mu}(s)\right)=M u^{\mu}
$$

So the average momentum of the two trajectories is simply a constant, just as one would expect for a classical free particle. Moreover, the angular momentum for the pair of trajectories about the center point of the Gaussian at world time $s=0$ is

$$
\begin{gathered}
M_{ \pm}^{\mu \nu}(s)=X_{B \pm}^{[\mu} V_{B \pm}^{\nu]}=u^{[\mu} X_{B \pm}^{\nu]}(0)\left[\frac{s^{2} \Gamma(\nu)^{2}}{\left(1+\Gamma(\nu)^{2} s^{2}\right)^{1 / 2}}-\left(1+\Gamma(\mu)^{2} s^{2}\right)^{1 / 2}\right] \\
M^{\mu \nu}(s)=\frac{1}{2}\left[X_{B+}^{[\mu} V_{B+}^{\nu]}+X_{B-}^{[\mu} V_{B-}^{\nu]}\right]=0
\end{gathered}
$$

So the combined orbital angular momentum of the pair of trajectories equally weighted is zero.

\section{Riemann sheet structure of $\mathbf{N}$ particle system}

Imagine an $\mathrm{N}$ particle system of non-interacting particles described by (1). Assume for simplicity that the wave function is a simple product for starters. Then, there will be in general two Riemann sheet trajectories per particle. This means that for $\mathrm{N}$ particles there will be $2^{N}$ Riemann sheets. For entangled states the situation is not clear, and will be left as a future research problem. 


\section{Spin - Modeling a Kerr-Newman particle off the real axis and riding along a Bohmian trajectory}

Up to this point we have been considering real trajectories, and therefore spinless Kerr-Newman particles, or Reissner-Nordström metric solutions 4 The Kerr-Newman particle in complex spacetime, and in a Lorentz frame in which it is at rest, is displaced from the real axis by a fixed amount $\vec{O}=i \vec{J} / m c$, where $J=|\vec{J}|$ is the angular momentum of the Kerr-Newman solution 22. This is the more interesting case, since most elementary charged particles (quarks and leptons) have spin. In our Gaussian case, if $u^{\mu} u_{\mu}=1$, then we can move to its rest frame with a single Lorentz boost. So we can obtain the imaginary part of a moving particle by Lorentz boosting $O^{\mu}=\{0, i \vec{J} / m c\}$ to a proper velocity $u$. $O^{\mu}(u)=\Lambda_{\nu}^{\mu}(u) O^{\nu}$. The natural thing to do is to add this boosted $O^{\mu}$ to $X_{B \pm}^{\mu}(0)$ in (40), but when we do this we run into poblems because then the displacement along the imaginary direction will grow and change with $\mathrm{s}$ in a way that will change its spin value. To see this, recall that for our Gaussian case this would imply

$$
X_{B \pm}^{\mu}(s)=u^{\mu} s \pm\left(X_{B}^{\mu}(0)+O^{\mu}(u)\right)\left(1+\Gamma(\mu)^{2} s^{2}\right)^{1 / 2}
$$

for large values of s the imaginary term proportional to $O^{\mu}(u)$ grows linearly in s. The spin angular momentum is therefore not constant, and also the particle does not remain close to the real hyperspace. This is inconsistent with the quantization of spin angular momentum. If $\Gamma(\mu)$ is small enough, and the particle lifetime is short then the present model might still be possible, but for an electron, as shown above (44), the spin would vary with $s$ in a physically unacceptable manner. Therefore this way of introducing spin cannot describe quantum electrons. Muons probably can't be described either this way. But quarks have a chance because they are never free, except in the quark gluon plasma. Vector Bosons $W^{+}, W^{-}$have very short lifetimes, and their measured $\mathrm{g}$ factor is also reasonably close to 2.042 as predicted by the Kerr-Newman theory. They are too short-lived for the variation of the imaginary part of their Bohmian trajectory to cause a problem.

There is another way to plausibly introduce spin. We can simply assume that the Bohmian trajectories for a free particle give the real part of the trajectory only, and that the effect of spin is simply to add a constant complex term to this real trajectory. This leads to the same current density as for the spinless case (75), with a modified electromagnetic potential. In the usual real case, the retarded potentials can be written

$$
A^{\mu}(x)=\int \frac{J^{\mu}\left(\vec{x}^{\prime}, x_{r}^{\prime 0}\right)}{\sqrt{\left(\vec{x}-\vec{x}^{\prime}\right)^{2}}} d^{3} x^{\prime}, x_{r}^{\prime 0}=x^{0}-\sqrt{\left(\vec{x}-\vec{x}^{\prime}\right)^{2}}
$$

${ }^{4}$ For elementary particles, these metrics have a naked singularity due to the electromagnetic field energy. If the particle becomes effectively an extended jellium by the GAN transformation, then this naked singularity would generally disappear. 
Now we would like to analytically continue this by $x^{\prime} \rightarrow x^{\prime}+O$. Let's start off with a real displacement, so all $O^{\mu}$ are real initially. Then the vector potential becomes

$$
A^{\mu}(x, O)=\int \frac{J^{\mu}\left(\vec{x}^{\prime}, x_{r}^{\prime 0}\right)}{\sqrt{\left(\vec{x}-\vec{x}^{\prime}-\vec{O}\right)^{2}}} d^{3} x^{\prime}, x_{r}^{\prime 0}=x^{0}-O^{0}-\sqrt{\left(\vec{x}-\vec{x}^{\prime}-\vec{O}\right)^{2}}
$$

where $x_{r}^{\prime 0}$ is the retarded time. In this form, the vector potential can be analytically continued to complex values of $O$, and then the projection onto real electromagnetic fields as in section 4 can be performed.

This model for spin is still too simple to lead to a description for a quantum electron as a Kerr-Newman particle. An explanation for the quantum behavior of spin is needed in this context. When one tries to make sense of the Dirac equation as a quantum wave equation, one is confronted with the zitterbewegung phenomenon. The Foldy-Wouthuysen transformation removes the zitterbewegung from the motion for positive energy states and produces a non-radiating current density 28]. One would think that a description of quantum fermions in the current framework should incorporate some explanation for zitterbewegung for Kerr-Newman particles. One such is given in [26] which is based on the classical Lorentz-Dirac equation embedded in complex space-time. Another model for zitterbwegung has been suggested by Burinskii, based on his gravitating bag model 17, 16. The bag structure gives internal structure to the particle, and it's no longer a simple point in complex space-time, but rather a closed loop string in space. This ring acts as a waveguide for massless particle excitations travelling the circumference of the ring, and this is the model for zitterbewegung [21. It also makes a connection with string theory which is an interesting way to obtain a connection with the Standard model of particle physics. It seems reasonable to try and couple the Bohmian picture with one of these zitterbewegung models. It's not obvious to me how to proceed though. So this task remains as a topic for future research.

\section{The non-relativistic Schrödinger equation}

It is straightforward to parallel the treatment provided here for the relativistic case for the the standard non-relativistic Schrödinger equation. For the Gaussian case we can use Holland's results (25) from [41. We again embed these trajectories in complex space-time. In the non-relativistic case, the trajectories are functions of the usual time variable $t$ rather than the world time $s$. Everything proceeds more or less as in the relativistic case. The retarded times are still multivalued, and the GAN continuation still generates all of the trajectories from a single trajectory. The non-radiating property holds as well for Schrödinger current [28].

\section{Interpretation of complex space-time}

A reasonable question to ask is "Don't we live in a real space-time universe?", and consequently "doesn't that rule out most of this paper?". The simplest answer I can give is - maybe we don't live in a real Minkowski space. Maybe we actually do live in complex space-time, i.e. a 4 dimensional complex manifold endowed with a Minkowski 
metric tensor, at least locally. Imagine that the universe is built up from point particles moving around but always very near to the real hyperspace, moving say along Bohmian trajectories and in some interaction with one another. Then all of our experimental apparatuses, being made up of such particles, are aware only of macroscopic distances in the real space-time which is given empirical prominence because all the matter is located there and large apparatuses are always therefore embedded in this 4-dimensional real subspace. For large objects, and for charged macro particles involving many elementary particles, I expect everything to look like ordinary classical electromagnetism in real space-time. All of the Bohmian trajectories for many particles will result in much averaging, and only the dominant retarded time for an extended macro particle will be found to contribute I suspect (or more honestly hope). When dealing with a single small elementary particle over small distances, I expect the corrections due to the complex space-time effects to become visible. What we call quantum mechanics might, in this fanciful picture, be the appearance of complex space time effects for microscopic objects. The fuzziness of quantum mechanics might be due to the extended particle nature of the electromagnetic fields produced, and to the multi-valued nature of trajectories and fields when small particles are being focused on. So, we think we live in a quantum world of 4 real dimensions, and our classical world is the large particle limit of this which obeys classical equations of motion, but maybe in reality we are living in a multi-valued but classical universe which consists of 4 complex dimensions, with the matter concentrated on the real hyperspace, and what we call quantum behavior, that we observe for small particles, is actually the effects of the complex space-time modifying the Liénard-Wiechert potentials and generating multi-valued fields and positions for small particles. I grant that this is a speculative and fanciful picture, but in fact it is in my mind when I think about these things.

There is another possibility though, and this is slightly different, but I think probably closer to what might be preferred by relativists. Remember that there is always a projection to real fields on a real hyperspace at the end of a calculation of the electromagnetic field produced by some complex trajectory (or real trajectory embedded complex space-time). So we always end up with real electromagnetic fields. We can simply assume that classical electromagnetism should include all of the roots that would be obtained by complex analytic continuation first with the resulting fields then projected down on the real hyperspace. This would then yield the same result as above where we treated the space as actually a complex manifold. This would still yield multi-valued fields and positions for particles.

This is the sense that I meant when I said in the introduction that I'm agnostic about whether the complex space-time was ontologically real or not.

\section{An analytic universe interpretation of quantum mechanics}

Maybe quantum mechanics is a description of a multi-Riemann-sheeted universe. Quantum superposition might be related to the GAN continuation which is a form of linear superposition of different Riemann sheets. It would need to be applied to the wave function itself, rather than to the trajecories, and so it might come about in a second quantization of the wave function treated as a dynamical field. Quantum mechanics is perfectly linear, as measured in many experiments [52,3,10. This is surprising. If quantum mechanics has some deep dynamical origin, how does it arise that linear superposition of wave functions is exactly correct? In the classical world linearity is 
always only approximate. The generalized analytic continuation based on a partition of unity argument gives exact linearity with absolutely no non-linear term. And yet, it arises from the mathematics of general relativity, electromagnetism, and Bohmian mechanics in complex space-time. One can start with the many worlds or Everett interpretation of quantum mechanics [33, and ask if the many worlds can be replaced by a many-Riemann-sheeted interpretation of quantum mechanics? Regarding the EinsteinRosen bridge and entanglement ideas [54,69, perhaps these too can be interpreted in terms of Riemann sheets. One might imagine that collapse of the wave function is related to an abrupt change in the weighting factors for different Riemann sheets as in the generalized analytic continuation idea, or perhaps in a change in the Riemann sheet structure itself. This is because a wave function collapse would cause a change in the Bohmian trajectories and their weighting functions and perhaps in their analytic Riemann sheet topology. The results of this paper together with [26] and the complex manifold techniques of general relativity seem to be suggesting such an analytic universe interpretation as a possibility.

\section{Conclusion}

If Kerr-Newman particles are coupled to the electromagnetic fields in complex spacetime, and are moving along Bohmian trajectories, then the trajectory equations become multi-valued with two or more Riemann sheets. If one accepts the generalized analytic continuation (GAN) proposal as valid, then for the Gaussian wave functions studied here, all the Bohmian trajectories can be continued into one another, except for a set of measure zero. This explains how such Kerr-Newman particles need not radiate electromagnetically. It also allows the point particle to act as an extended charge distribution or jellium which would generally have a finite electrostatic energy. The hydrodynamic or jellium model makes an experimentally testable prediction involving bremsstrahlung as well 25. In the standard Bohmian interpretation, one is not allowed to couple the charged particles in this way. So these results are an application of Bohmian mechanics to a hybrid interpretation combining the Kerr-Newman complex space-time techniques with Bohmian mechanics. The results here in no way detract from the standard Bohmian interpretation, but add a possible new application for Bohmian mechanics.

Although metric nonlinearities are ignored in this paper, the complex space-time embedding is not ignored, and the motivation for this embedding comes from general relativity. Therefore, the multi-sheeted and multi-valued trajectories and resulting fields are also a direct consequence of general relativity that cannot be ignored, even in the very weak gravity limit. Thus we have a flat space theory in which general relativity is playing a very major role, and in fact it's offering potential explanations for some of the deepest mysteries of quantum mechanics. It also leads to a way to avoid the infinite self-energy of a static point particle in classical electromagnetism by making a point charge behave as an extended particle.

For multi-particle systems the multiplicity of Riemann sheets increases exponentially. These results beg the question - Can an interpretation of quantum mechanics be developed that is based on this multi-sheeted structure, in a sense mapping the spirit of the many-worlds or Everett interpretation 33] into a single analytic complex world manifold with many Riemann sheets? 
Acknowledgements I would like to thank the two reviewers for this paper who provided valuable insights and suggestions. I would also like to thank Ezra Newman for his helpful correspondence.

\section{References}

1. T. M. Adamo, C. Kozameh, and E. T. Newman. Null Geodesic Congruences, Asymptotically-Flat Spacetimes and Their Physical Interpretation. Living Reviews in Relativity (www.livingreviews.org/lrr-2009-6), 12(6), 2009.

2. I. Aharonovich and L. P. Horwitz. Radiation-reaction in classical off-shell electrodynamics. I. The above mass-shell case. Journal of Mathematical Physics, 53(3):032902-032902-29, March 2012.

3. Markus Arndt and Klaus Hornberger. Testing the limits of quantum mechanical superpositions. Nature Physics, 10(4):271-277, April 2014

4. Guido Bacciagaluppi and Antony Valentini. Quantum Theory at the Crossroads: Reconsidering the 1927 Solvay Conference. Cambridge University Press, Cambridge, UK ; New York, 1 edition edition, November 2009.

5. A. O. Barut. Quantum-Electrodynamics Based on Self-Energy. Physica Scripta, 1988(T21):18, 1988.

6. A. O. Barut and Jonathan P. Dowling. Self-field quantum electrodynamics: The two-level atom. Physical Review A, 41(5):2284-2294, March 1990.

7. A. O. Barut and Jonathan P. Dowling. QED Based on Self-Fields: A Relativistic Calculation of g-2. Zeitschrift für Naturforschung A, 44(11):1051-1056, 2014.

8. null Barut and null Dowling. Interpretation of self-field quantum electrodynamics. Physical Review. A, Atomic, Molecular, and Optical Physics, 43(7):4060, April 1991.

9. Carl M. Bender, Dorje C. Brody, and Hugh F. Jones. Complex Extension of Quantum Mechanics. Physical Review Letters, 89(27):270401, December 2002.

10. Iwo Bialynicki-Birula. On the linearity of the Schrödinger equation. Brazilian Journal of Physics, 35(2A):211-215, June 2005.

11. David Bohm and Basil J. Hiley. The Undivided Universe: An Ontological Interpretation of Quantum Theory. Routledge, London; New York, revised ed. edition edition, March 1995.

12. A. Burinskii. Kerr spinning particle, strings, and superparticle models. Physical Review D, 57(4):2392, 1998.

13. A. Burinskii. The Dirac-Kerr electron. July 2005.

14. A. Burinskii. Kerr Geometry as Space-Time Structure of the Dirac Electron. arxiv.org 0712.0577, 2007.

15. A. Burinskii. The dirac-kerr-newman electron. Gravitation and Cosmology, 14:109-122, 2008 .

16. A. Burinskii. Emergence of the Dirac equation in the solitonic source of the Kerr spinning particle. Gravitation and Cosmology, 21(1):28-34, January 2015.

17. A. Burinskii. Gravitating lepton bag model. Journal of Experimental and Theoretical Physics, 121(2):194-205, August 2015.

18. A. Ya Burinskii. Microgeon with a Kerr metric. Soviet Physics Journal, 17(8):1068-1071, August 1974.

19. Alexander Burinskii. Regularized Kerr-Newman Solution as a Gravitating Soliton. arXiv:1003.2928, March 2010. J. Phys. A: Math. Theor. 43 (2010) 392001.

20. Alexander Burinskii. Gravitational strings beyond quantum theory: Electron as a closed string. arXiv:1109.3872, September 2011.

21. Alexander Burinskii. Kerr-newman electron as spinning soliton. International Journal of Modern Physics A, 29(26):1450133, September 2014.

22. Alexander Burinskii and Giulio Magli. Kerr-Schild approach to the boosted Kerr solution. Physical Review D, 61(4):044017, January 2000.

23. Chia-Chun Chou and Robert Wyatt. Complex-extended Bohmian mechanics. The Journal of Chemical Physics, 132(13):134102, April 2010.

24. Ron Cowen. The quantum source of space-time. Nature News, 527(7578):290, November 2015. 
25. Mark Davidson. Predictions of the hydrodynamic interpretation of quantum mechanics com-pared with quantum electrodynamics for low energy bremsstrahlung. Annales de la Fondation Louis de Broglie, 29(4):661-680, 2004.

26. Mark Davidson. The Lorentz-Dirac equation in complex space-time. General Relativity and Gravitation, 44(11):2939-2964, November 2012. arXiv: 1109.4923.

27. Mark Davidson. A study of the Lorentz-Dirac equation in complex space-time for clues to emergent quantum mechanics. Journal of Physics: Conference Series, 361(1):012005, 2012.

28. Mark P. Davidson. Quantum wave equations and non-radiating electromagnetic sources. Annals of Physics, 322(9):2195-2210, September 2007. arXiv: quant-ph/0606245.

29. Maaneli Derakhshani. A Suggested Answer To Wallstrom's Criticism: Zitterbewegung Stochastic Mechanics I. arXiv:1510.06391 [quant-ph], October 2015. arXiv: 1510.06391.

30. Detlef Dürr, Sheldon Goldstein, Travis Norsen, Ward Struyve, and Nino Zanghì. Can Bohmian mechanics be made relativistic? Proc.Roy.Soc.Lond., A470:20130699, 2013.

31. Detlef Dürr, Sheldon Goldstein, and Nino Zanghì. Quantum Physics Without Quantum Philosophy. Springer, Heidelberg ; New York, 2013 edition edition, November 2012.

32. A. Einstein and N. Rosen. The Particle Problem in the General Theory of Relativity. Physical Review, 48(1):73-77, July 1935.

33. Hugh Everett. The Theory of the Universal Wave function. In The Many-Worlds Interpretation of Quantum Mechanics, edited by Bryce S. Dewitt and Neill Graham, pages 3-140. Princeton University Press, Princeton, 1973.

34. John R. Fanchi. Quantum Potential in Relativistic Dynamics. Foundations of Physics, 30(8):1161-1189, August 2000.

35. V. Fock. Die Eigenzeit in der klassischen und in der Quantenmechanik. Phys. Z. Sowjetunion, 12:404-425, 1937.

36. Daniel M. Greenberger. Theory of Particles with Variable Mass. I. Formalism. Journal of Mathematical Physics, 11(8):2329-2340, August 1970.

37. Daniel M. Greenberger. Theory of Particles with Variable Mass. II. Some Physical Consequences. Journal of Mathematical Physics, 11(8):2341-2347, August 1970.

38. Daniel M. Greenberger. Some useful properties of a theory of variable mass particles. Journal of Mathematical Physics, 15(4):395, 1974.

39. Daniel M. Greenberger. Wavepackets for particles of indefinite mass. Journal of Mathematical Physics, 15(4):406, 1974.

40. David Hestenes. The zitterbewegung interpretation of quantum mechanics. Foundations of Physics, 20(10):1213-1232, October 1990.

41. Peter R. Holland. The Quantum Theory of Motion: An Account of the de Broglie-Bohm Causal Interpretation of Quantum Mechanics. Cambridge University Press, Cambridge, revised ed. edition edition, February 1995.

42. Barry R. Holstein. How large is the "natural" magnetic moment? American Journal of Physics, 74(12):1104-1111, November 2006.

43. Lawrence Horwitz. A Statistical Mechanical Model for Mass Stability in the SHP Theory. arXiv:1607.03742 [physics], July 2016. arXiv: 1607.03742.

44. Lawrence P. Horwitz. Relativistic Quantum Mechanics. Springer Netherlands, Dordrecht, 2015.

45. John David Jackson. Classical electrodynamics, 3rd Ed. Wiley, 1999.

46. A. Kyprianidis. Particle trajectories in relativistic quantum mechanics. Physics Letters A, 111:111-116, September 1985.

47. A. Kyprianidis. Scalar time parametrization of relativistic quantum mechanics: The covariant Schrödinger formalism. Physics Reports, 155(1):1-27, November 1987.

48. M. C. Land. Pre-Maxwell Electrodynamics. Foundations of Physics, 28(9):1479-1487, September 1998.

49. M. C. Land and L. P. Horwitz. Green's functions for off-shell electromagnetism and spacelike correlations. Foundations of Physics, 21(3):299-310, March 1991.

50. Martin Land. Speeds of light and mass stability in Stueckelberg-Horwitz-Piron electrodynamics. arXiv:1604.01638 [hep-th, physics:physics], April 2016. arXiv: 1604.01638.

51. Martin Land and Lawrence P. Horwitz. Offshell quantum electrodynamics. Journal of Physics: Conference Series, 437(1):012011, 2013.

52. A. J. Leggett. Testing the limits of quantum mechanics: motivation, state of play, prospects. Journal of Physics: Condensed Matter, 14(15):R415, 2002.

53. D. Lynden-Bell. A magic electromagnetic field. In Stellar Astrophysical Fluid Dynamics, pages 369-375. 2003. 
54. J. Maldacena and L. Susskind. Cool horizons for entangled black holes. Fortschritte der Physik, 61(9):781-811, September 2013.

55. E. T. Newman. Complex coordinate transformations and the Schwarzschild-Kerr metrics. Journal of Mathematical Physics, 14:774, 1973.

56. E. T. Newman. Heaven and its properties. General Relativity and Gravitation, 7(1):107111, January 1976

57. E. T. Newman, E. Couch, K. Chinnapared, A. Exton, A. Prakash, and R. Torrence. Metric of a Rotating, Charged Mass. Journal of Mathematical Physics, 6(6):918, 1965.

58. Ezra T. Newman. Maxwell's equations and complex Minkowski space. Journal of Mathematical Physics, 14(1):102, 1973.

59. Ezra T. Newman. Private communication. 2017.

60. C. L. Pekeris and K. Frankowski. The electromagnetic field of a Kerr-Newman source. Physical Review A, 36(11):5118, 1987.

61. Roger Penrose. The Road to Reality: A Complete Guide to the Laws of the Universe. Vintage, New York, reprint edition edition, January 2007.

62. Roger Penrose. Fashion, Faith, and Fantasy in the New Physics of the Universe. Princeton University Press, Princeton Oxford, September 2016.

63. Bill Poirier. Flux continuity and probability conservation in complexified Bohmian mechanics. Physical Review A, 77(2):022114, February 2008.

64. Eric Poisson. A Relativist's Toolkit: The Mathematics of Black-Hole Mechanics. Cambridge University Press, Cambridge; New York, N.Y., 1 edition edition, November 2007.

65. Mark Van Raamsdonk. Building up spacetime with quantum entanglement. General Relativity and Gravitation, 42(10):2323-2329, October 2010.

66. Carlo Rovelli. Relational quantum mechanics. International Journal of Theoretical Physics, 35(8):1637-1678, August 1996.

67. Tilman Sauer. Einstein's unified field theory program. In The Cambridge companion to Einstein. Cambridge University Press, New York, NY, 2014.

68. E. Stueckelberg. La signification du temps propre en mécanique ondulatoire. Helv. Phys. Acta, 14:322-323, 1941.

69. Leonard Susskind. Dear Qubitzers, GR=QM. arXiv:1708.03040 [hep-th], August 2017. arXiv: 1708.03040 .

70. Mark Swanson. Transition elements for a non-Hermitian quadratic Hamiltonian. Journal of Mathematical Physics, 45(2):585-601, January 2004.

71. Gerard 't Hooft. The Cellular Automaton Interpretation of Quantum Mechanics. arXiv:1405.1548 [quant-ph], May 2014. arXiv: 1405.1548.

72. Erik Verlinde. Emergent Gravity and the Dark Universe. SciPost Physics, 2(3):016, May 2017. arXiv: 1611.02269.

73. Steven Weinberg. Collapse of the state vector. Physical Review A, 85(6):062116, June 2012 . 